\title{
Memory effect of the pp waves with torsion
}

\author{
B. Cvetkovića ${ }^{\mathrm{a}}$ D. Simić ${ }^{\mathrm{b}}$ \\ Institute of Physics, University of Belgrade, Pregrevica 118, 11080 Belgrade, Serbia
}

Received: 3 January 2022 / Accepted: 29 January 2022 / Published online: 9 February 2022

(C) The Author(s) 2022

\begin{abstract}
We analyse the motion of test particles in the spacetime of the plane-fronted (pp) waves with torsion in four-dimensions. We conclude that there is a velocity memory effect in the direction of advanced time and along radial direction, while we have rotation of particles in angular direction. The velocity memory effect in the aforementioned directions is severely affected by the value of the tordion mass and probably it is not observable. A very interesting, probably observable effect, steams from the rotation, which is insensitive to the tordion mass.
\end{abstract}

\section{Introduction}

When a gravitational wave passes through a system of test particles it induces an observable disturbance of the system [1-4]. In other words a system remembers that a wave passed, and for this reason it is known as the memory effect.

There are two possible outcomes when a wave passes, disregarding the trivial possibility that everything is translated or boosted in the same way which is unobservable. The first scenario is that relative velocity of test particles is zero while they suffer a permanent displacement depending on their initial conditions. This is known as the displacement memory effect [1-4], for the results on nonlinear contribution to the memory effect see $[5,6]$. The appearance of displacement memory effect is questioned in [7-9], where authors concluded that test particles will have non-zero relative velocity. This variation of the memory effect is known as the velocity memory effect, for recent development see [10-12].

All of the previously mentioned results are obtained in the framework of general relativity and memory effect is not much investigated beyond it. Some of the results, known to the authors, are memory effect for massive graviton investigated in Ref. [13] and memory effect for pp waves in General

\footnotetext{
a e-mail: cbranislav@ipb.ac.rs

be-mail: dsimic@ipb.ac.rs (corresponding author)
}

relativity [14], while, the memory effect of the gravitational waves with torsion in the Poincare gauge theory (PGT) has been investigated only in three-dimensions in Ref. [15].

The aim of this paper is to fill the gap in the literature, namely to extend results about the memory effect to the gravitational waves with torsion in four-dimensions.

Basic dynamical variables in PGT [16-19] are the tetrad field $b^{i}$ and the Lorentz connection $\omega^{i j}=-\omega^{j i}$ (1-forms), and the associated field strengths are the torsion $T^{i}=d b^{i}+$ $\omega^{i}{ }_{k} \wedge b^{k}$ and the curvature $R^{i j}=d \omega^{i j}+\omega^{i}{ }_{k} \wedge \omega^{k j}$ forms). By construction, PGT is characterized by a RiemannCartan geometry of spacetime, and its physical content is directly related to the existence of mass and spin as basic characteristics of matter at the microscopic level. General PGT Lagrangian $L_{G}$ is at most quadratic in the field strengths. The number of independent (parity invariant) terms in $L_{G}$ is nine, which makes the corresponding dynamical structure rather complicated.

The paper is organized as follows. First, we review the gravitational pp wave solutions with torsion in fourdimensions. After that, we derive the geodesic equations in this pp wave spacetime. We finally numerically solve the geodesic equations.

Our conventions are as follows. The Latin indices $(i, j, \ldots)$ refer to the local Lorentz (co)frame and run over $(0,1,2,3)$, $b^{i}$ is the tetrad (1-form), $h_{i}$ is the dual basis (frame), such that $\left.h_{i}\right\lrcorner b^{k}=\delta_{k}^{i}$; the volume 4-form is $\hat{\epsilon}=b^{0} \wedge b^{1} \wedge b^{2} \wedge$ $b^{3}$, the Hodge dual of a form $\alpha$ is ${ }^{\star} \alpha$, with ${ }^{\star} 1=\hat{\epsilon}$, totally antisymmetric tensor is defined by ${ }^{\star}\left(b^{i} b^{j} b^{k} b^{l}\right)=\varepsilon^{i j k l}$ and normalized to $\varepsilon_{0123}=+1$; the exterior product of forms is implicit in all expressions.

\section{Review of the pp waves}

In this section, we give an overview of 4D pp waves in PGT. For details see [20]. 


\section{$2.1 \mathrm{pp}$ waves without torsion}

\subsubsection{Geometry}

In local coordinates $x^{\mu}=(u, v, y, z)$, the metric of the pp waves is of the form

$d s^{2}=d u(H d u+2 d v)-\left(d y^{2}+d z^{2}\right)$,

where the unknown metric function $H=H(u, y, z)$ is to be obtained from the field equations. The advanced time $v$ is an affine parameter along the null geodesics $x^{\mu}=x^{\mu}(v)$, and $u$ is retarded time such that $u=$ const. are the spacelike surfaces parameterized by $x^{\alpha}=(y, z)$. Since the null vector $\xi=\xi(u) \partial_{v}$ is orthogonal to these surfaces, they are regarded as wave surfaces, and $\xi$ is the null direction (ray) of the wave propagation.

We choose the tetrad field (coframe) to be of the form

$$
\begin{aligned}
& b^{0}:=d u, \quad b^{1}:=\frac{H}{2} d u+d v, \\
& b^{2}:=d y, \quad b^{3}:=d z,
\end{aligned}
$$

so that $d s^{2}=\eta_{i j} b^{i} \otimes b^{j}$, where $\eta_{i j}$ is the half-null Minkowski metric:

$\eta_{i j}=\left(\begin{array}{cccc}0 & 1 & 0 & 0 \\ 1 & 0 & 0 & 0 \\ 0 & 0 & -1 & 0 \\ 0 & 0 & 0 & -1\end{array}\right)$.

The corresponding dual frame $h_{i}$ is given by

$h_{0}=\partial_{u}-\frac{H}{2} \partial_{v}, \quad h_{1}=\partial_{v}, \quad h_{2}=\partial_{y}, \quad h_{3}=\partial_{z}$.

For the coordinates $x^{\alpha}=(y, z)$ on the wave surface, we have:

$x^{c}=b_{\alpha}^{c} x^{\alpha}=(y, z), \quad \partial_{c}=h_{c}{ }^{\alpha} \partial_{\alpha}=\left(\partial_{y}, \partial_{z}\right)$,

where $c=2,3$. After introducing the notation $i=(A, a)$, where $A=0,1$ and $a=(2,3)$, one can find the compact form of the Riemannian connection $\omega^{i j}$ :

$$
\omega^{A c}=\frac{1}{2} k^{A} b^{0} \partial^{c} H,
$$

where $k^{i}=(0,1,0,0)$ is a null propagation vector, $k^{2}=0$.

The above connection defines the Riemannian curvature $R^{i j}=d \omega^{i j}+\omega^{i}{ }_{m} \omega^{m j}$; for $i<j$, it is given by

$R^{i j}=2 b^{0} k^{[i} Q^{j]}$

where $Q^{c}$ is a 1-form introduced by Obukhov [21],

$$
\begin{aligned}
Q^{2} & =\frac{1}{2} \partial_{y y} H b^{2}+\frac{1}{2} \partial_{y z} H b^{3}, \\
Q^{3} & =\frac{1}{2} \partial_{z z} H b^{3}+\frac{1}{2} \partial_{y z} H b^{2} .
\end{aligned}
$$

The Ricci 1-form $\left.R i c^{i}:=h_{m}\right\lrcorner R i c^{m i}$ is given by

$$
\begin{aligned}
& R i c^{i}=b^{0} k^{i} Q, \\
& \left.Q=h_{c}\right\lrcorner Q^{c}=\frac{1}{2}\left[\partial_{y y} H+\partial_{z z} H\right],
\end{aligned}
$$

and the scalar curvature $\left.R:=h_{i}\right\lrcorner R i c^{i}$ vanishes.

\subsection{2 pp waves in $G R$}

Starting with the action $I_{0}=-\int d^{4} x a_{0} R$, one can derive the GR field equations in vacuum:

$2 a_{0} G^{n}{ }_{i}=0$,

where $G^{n}{ }_{i}$ is the Einstein tensor. As a consequence, the metric function $H$ must obey

$\partial_{y y} H+\partial_{z z} H=0$.

There is a simple solution of these equations,

$H_{c}=A(u)+B_{\alpha}(u) x^{\alpha}$,

for which $Q^{a}$ vanishes. This solution is trivial (or pure gauge), since the associated curvature takes the background form, $R^{i j}=0$.

\section{2 pp waves with torsion}

\subsubsection{Geometry of the ansatz}

We assume that the form of the triad field (2.2) remains unchanged, while looking at the Riemannian connection (2.3), one can notice that its radiation piece appears only in the $\omega^{1 c}$ components:

$\left(\omega^{1 c}\right)^{R}=\frac{1}{2}\left(h^{c \alpha} \partial_{\alpha} H\right) b^{0}$.

This motivates us to construct a new connection by applying the rule

$\frac{1}{2} \partial_{\alpha} H \rightarrow \frac{1}{2} \partial_{\alpha} H+K_{\alpha}, \quad K_{\alpha}=K_{\alpha}(u, y, z)$,

where $K_{\alpha}$ is the component of the 1 -form $K=K_{\alpha} d x^{\alpha}$ on the wave surface. Thus, the new form of $\left(\omega^{i j}\right)^{R}$ reads

$\left(\omega^{i c}\right)^{R}:=k^{i} h^{c \alpha}\left(\frac{1}{2} \partial_{\alpha} H+K_{\alpha}\right) b^{0},$.

The geometric content of the new connection is found by calculating the torsion:

$T^{i}=\nabla b^{i}+\omega_{m}^{i} b^{m}=k^{i} b^{0}\left(b^{2} K_{y}+b^{3} K_{z}\right)=k^{i} b^{0} b^{c} K_{c}$.

The only nonvanishing irreducible piece of $T^{i}$ is ${ }^{(1)} T^{i}$.

The new connection modifies also the curvature, so that its radiation piece becomes

$\left(R^{1 c}\right)^{R}=k^{1} b^{0} \Omega^{c}, \quad \Omega^{c}:=Q^{c}+\Theta^{c}$, 
where the term $\Theta^{c}$ that represents the contribution of torsion is given by

$$
\Theta^{2}=\partial_{y} K_{y} b^{2}-\partial_{z} K_{y} b^{3}, \quad \Theta^{3}=\partial_{z} K_{z} b^{3}-\partial_{y} K_{z} b^{2} .
$$

The covariant form of the curvature reads

$$
R^{i j}=2 b^{0} k^{[i} \Omega^{j]},
$$

and the Ricci curvature takes the form

$$
\left.R i c^{i}=b^{0} k^{i} \Omega, \quad \Omega:=h_{c}\right\lrcorner \Omega^{c}=Q+\Theta .
$$

The torsion has no influence on the scalar curvature and it again vanishes. Thus, our ansatz defines a RC geometry of spacetime.

\subsubsection{Massive torsion waves}

The irreducible decomposition of the curvature implies (see [20])

$$
{ }^{(3)} R_{i j}=0, \quad{ }^{(5)} R_{i j}=0, \quad{ }^{(6)} R_{i j}=0
$$

whereas the remaining pieces ${ }^{(n)} R^{i j}$ are defined by their nonvanishing components as

$$
\begin{aligned}
& { }^{(2)} R^{1 c}=\frac{1}{2}{ }^{\star}\left(\Psi^{1} b^{c}\right), \quad{ }^{(4)} R^{1 c}=\frac{1}{2}\left(\Phi^{1} b^{c}\right), \\
& { }^{(1)} R^{1 c}=b^{0}\left(\Omega^{(c e)}-\frac{1}{2} \eta^{c e} \Omega\right) b_{e},
\end{aligned}
$$

where the 1 -forms $\Phi^{i}$ and $\Psi^{i}$ are given by

$$
\begin{aligned}
& \Phi^{i}=k^{i} b^{0}(Q+\Theta), \quad \Theta=\partial_{y} K_{y}+\partial_{z} K_{z}, \\
& \Psi^{i}=X^{i}=-k^{i} b^{0} \Sigma, \quad \Sigma=\partial_{z} K_{y}-\partial_{y} K_{z} .
\end{aligned}
$$

Having found ${ }^{(1)} T_{i}$ and ${ }^{(n)} R_{i j}$, we obtain the following form of the two PGT field equations [20]:

$$
\begin{aligned}
& (1 \mathrm{ST}) \Lambda=0, \quad a_{1} \Theta-a_{0}(Q+\Theta)=0, \\
& (2 \mathrm{ND})-\left(b_{2}+b_{1}\right)\left(\nabla \Psi^{1}\right) b^{2}-\left(b_{4}+b_{1}\right)\left(\nabla \Phi^{1}\right) b^{3} \\
& \quad-2\left(a_{0}-A_{1}\right) T^{1} b^{3}=0, \\
& \quad-\left(b_{2}+b_{1}\right)\left(\nabla \Psi^{1}\right) b^{3}+\left(b_{4}+b_{1}\right)\left(\nabla \Phi^{1}\right) b^{2} \\
& \quad+2\left(a_{0}-A_{1}\right) T^{1} b^{2}=0 .
\end{aligned}
$$

Leaving (1ST) as it is, (2ND) can be given a more clear structure as follows:

$$
\begin{aligned}
& \left(\partial_{y y}+\partial_{z z}\right) \Theta-m_{2^{+}}^{2} \Theta=0, \quad m_{2^{+}}^{2}:=\frac{2 a_{0}\left(a_{0}-a_{1}\right)}{a_{1}\left(b_{1}+b_{4}\right)}(2.4 \mathrm{a}) \\
& \left(\partial_{y y}+\partial_{z z}\right) \Sigma-m_{2^{-}}^{2} \Sigma=0, \quad m_{2^{-}}^{2}:=\frac{2\left(a_{0}-a_{1}\right)}{b_{1}+b_{2}} .(2.4 \mathrm{~b})
\end{aligned}
$$

The parameters $m_{2^{ \pm}}^{2}$ have a simple physical interpretation. They represent masses of the spin- $2^{ \pm}$torsion modes with respect to the $M_{4}$ background [22,23], $\bar{m}_{2^{+}}^{2}=\frac{2 a_{0}\left(a_{0}-a_{1}\right)}{a_{1}\left(b_{1}+b_{4}\right)}, \quad \bar{m}_{2^{-}}^{2}=\frac{2\left(a_{0}-a_{1}\right)}{b_{1}+b_{2}}$.

In $M_{4}$, the physical torsion modes are required to satisfy the conditions of no ghosts (positive energy) and no tachyons (positive $m^{2}$ ) [22-24]. However, for spin-2 ${ }^{+}$and spin-2modes, the requirements for the absence of ghosts, given by the conditions $b_{1}+b_{2}<0$ and $b_{1}+b_{4}>0$, do not allow for both $m^{2}$ to be positive. Hence, only one of the two modes can exist as a propagating mode (with finite mass), whereas the other one must be "frozen" (infinite mass).

Important point to be noted is that the two spin-2 sectors have very different dynamical structures.

- In the spin-2- $2^{-}$sector, the infinite mass of the spin- $2^{+}$ mode implies $\Theta=0$, while (1ST) gives $Q=0$, which is nothing other than the GR field equation for metric. Consequently, the presence of torsion has no influence on the metric.

- In the spin- $2^{+}$sector, the infinite mass of the spin-2mode leads to $\Sigma=0$, whereas (1ST) gives that $Q$ is proportional to $\Theta$, with $\Theta \neq 0$. Leading to the conclusion that the torsion function $\Theta$ has a decisive dynamical influence on the metric.

We shall focus our attention on the spin- $2^{+}$sector, where the metric appears to be a genuine dynamical effect of PGT.

\subsubsection{Solutions in the spin- $2^{+}$sector}

After introducing polar coordinates $y=\rho \cos \varphi, z=$ $\rho \sin \varphi$, Eq. (2.4a) takes the form

$$
\left(\frac{\partial^{2}}{\partial \rho^{2}}+\frac{1}{\rho} \frac{\partial}{\partial \rho}+\frac{1}{\rho^{2}} \frac{\partial^{2}}{\partial \varphi^{2}}\right) \Theta-m^{2} \Theta=0 .
$$

Looking for a solution of $\Theta$ in the form of a Fourier expansion,

$\Theta=\sum_{n=0}^{\infty} \Theta_{n}(\rho)\left(c_{n} e^{i n \varphi}+\bar{c}_{n} e^{-i n \varphi}\right)$,

we obtain:

$\Theta_{n}^{\prime \prime}+\frac{1}{\rho} \Theta_{n}^{\prime}-\left(\frac{n^{2}}{\rho^{2}}+m^{2}\right) \Theta_{n}=0$,

where prime denotes $d / d \rho$.

The general solution of Eq. (2.5b) has the form

$\Theta_{n}=c_{1 n} J_{n}(-i m \rho)+c_{2 n} Y_{n}(-i m \rho), \quad n=0,1,2, \ldots$

where $J_{n}$ and $Y_{n}$ are Bessel functions of the 1st and 2nd kind, respectively. 


\subsubsection{Solutions for the metric function $\boldsymbol{H}$}

For a given $\Theta$, the first PGT field equation $a_{0} Q=\left(a_{1}-a_{0}\right) \Theta$, with $Q$ defined in (2.5), represents a differential equation for the metric function $H$ :

$\left(\partial_{y y}+\partial_{z z}\right) H=\frac{2\left(a_{1}-a_{0}\right)}{a_{0}} \Theta$.

This is a second order, linear nonhomogeneous differential equation, and its general solution can be written as

$H=H^{h}+H^{p}$,

where $H^{h}$ is the general solution of the homogeneous equation, and $H^{p}$ a particular solution of (2.7). One finds that there is a simple particular solution for $H$ :

$H^{p}=\sigma V, \quad \sigma=\frac{2\left(a_{1}-a_{0}\right)}{m^{2} a_{0}}$.

On the other hand, $H^{h}$ coincides with the general vacuum solution o $\mathrm{GR}_{\Lambda}$, see (2.7). Since our idea is to focus on the genuine torsion effect on the metric, we choose $H^{h}=0$ and adopt $H^{p}$ as the most interesting PGT solution for the metric function $H$. Thus, we have

$H_{n}=\sigma \Theta_{n}$.

The solutions for torsion functions are given in Appendix A.

\section{Geodesic motion}

In this section we shall examine the geodesic motion of particles in the field of the massive gravitational wave with torsion. We shall consider the motion of spinless particles in a gravitational field, which follow geodesic lines. It is known that torsion affects the motion of the particles with spin by causing its precession $[19,25]$. However, the gravitational waves with torsion, which we are considering are intrinsically different from the well-known spherically symmetric (static or stationary) solutions of PGT [26,27] (for review see [28]). The metric of these spherically symmetric solutions is "independent" of torsion in the sense that it represents Schwarzschild (or Schwarzschild AdS, Kerr etc.) metric and the motion of spinless particles is not affected by the presence torsion. For the gravitational wave solution (2.5), metric crucially depends on torsion, as we noted in the previous section.

Christoffel connection The non-vanishing components of Christoffel connection in polar coordinates are given by

$$
\begin{aligned}
& \tilde{\Gamma}_{u u}^{v}=\frac{1}{2} \partial_{u} H, \quad \tilde{\Gamma}_{u \rho}^{v}=\frac{1}{2} H^{\prime}, \quad \tilde{\Gamma}_{u \varphi}^{v}=\frac{1}{2} \partial_{\varphi} H, \\
& \tilde{\Gamma}_{u u}^{\rho}=\frac{1}{2} H^{\prime}, \quad \tilde{\Gamma}_{\varphi \varphi}^{\rho}=-\rho, \\
& \tilde{\Gamma}_{u u}^{\varphi}=\frac{1}{2 \rho^{2}} \partial_{\varphi} H, \quad \tilde{\Gamma}_{\rho \varphi}^{\varphi}=\frac{1}{\rho},
\end{aligned}
$$

where $H^{\prime}:=\partial_{\rho} H$.

Let us mention that we shall consider the solution with non-trivial contribution to metric function (and consequently Christoffel connection) stemming from the presence of torsion.

Geodesic equations. The geodesic equation for $u$ takes the expected form

$\frac{d^{2} u}{d \lambda^{2}}=\ddot{u}=0$.

Therefore without the loss of generality we can assume $u \equiv$ $\lambda$.

The equation for $v, \rho$ and $\varphi$ are given by:

$\ddot{v}+\frac{1}{2} \partial_{u} H+H^{\prime} \dot{\rho}+\partial_{\varphi} H \dot{\varphi}=0$.

$\ddot{\rho}+\frac{1}{2} H^{\prime}-\rho \dot{\varphi}^{2}=0$,

$\ddot{\varphi}+\frac{1}{2 \rho^{2}} \partial_{\varphi} H+\frac{2}{\rho} \dot{\rho} \dot{\varphi}=0$.

We shall solve the geodesic equations numerically, but let us first make some reasonable simplifications.

First, $v$ appears only as a second derivative because $H$ is independent of it. Consequently, we have a shift symmetry

$v \rightarrow v+c_{0}+c_{1} u$,

which means that initial conditions at time $u_{i}$ can be chosen as

$v\left[u_{i}\right]=v^{\prime}\left[u_{i}\right]=0$.

Second, in the metric function $H$ there is a factor

$\sigma=\frac{2\left(a_{1}-a_{0}\right)}{m^{2} a_{0}}$

where $a_{0}=\frac{1}{16 \pi G}$ is coupling constant of general relativity and $a_{1}$ corresponds to correction in the action stemming from torsion. Experimental results suggest that $a_{1}$ is much smaller than $a_{0}$ so we can approximate

$\sigma \approx-\frac{2}{m^{2}}$.

Also, we can introduce reduced variables

$\mathrm{v}=m^{2} v, r=m \rho$,

while $\varphi$ remains the same. In these variables geodesic equations do not have explicit dependence on $m$ and have more suitable form for numerical calculations.

\subsection{Memory effect}

We have one more unknown in geodesic equations and that is the form of functions $c_{1 n}$ and $c_{2 n}$. We expect that their exact form is not specially important, as long as they sufficiently fast tend to zero at infinity. But, we encountered numerical 

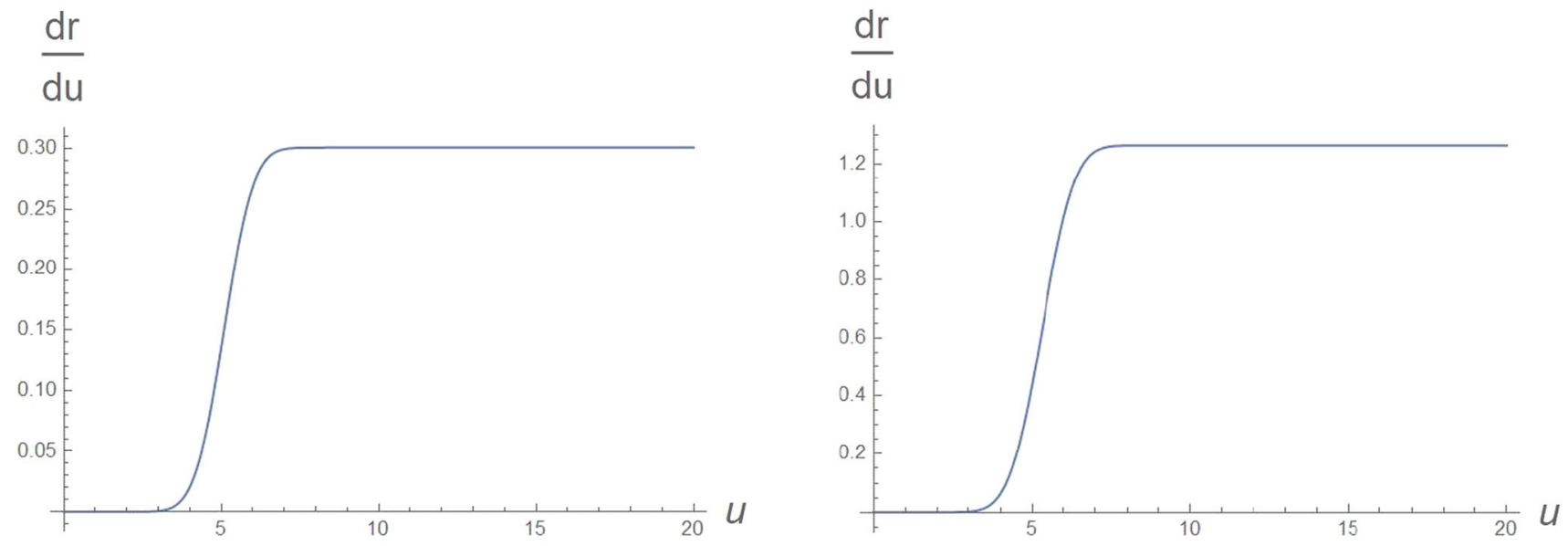

Fig. 1 The plot for the particle velocity $\dot{r}$ for $r[0]=1$ and $r[0]=2$
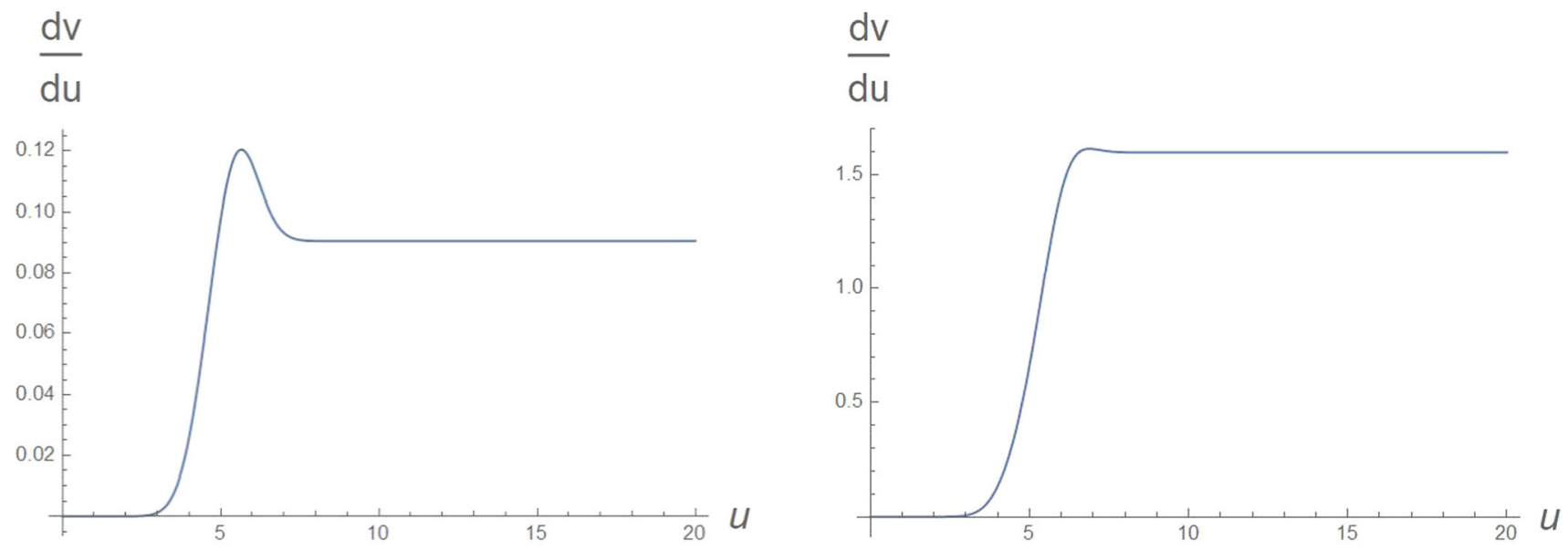

Fig. 2 The plot for the particle velocity $\dot{\mathrm{v}}$ for $r[0]=1$ and $r[0]=2$

problems because for polynomial fall-off the software cannot handle the computational complexity. Because, of this problem we decided to focus to Gaussian form of functions, more precisely to the form $e^{-(u-5)^{2}}$. For the initial time we chose $u=0$. As we already noted the initial conditions for $\tilde{v}$ are

$v[0]=\dot{v}[0]=0$,

and we assume that particle is initially at rest

$\dot{\rho}[0]=\dot{\varphi}[0]=0$.

So, the only variable inputs are $\rho[0]$ and $\varphi[0]$ as well as the modes $c_{1 n}$ and $c_{2 n}$ we are including.

Mode $J_{0}$. In this case we set $H=J_{0}(-i r) e^{-(u-5)^{2}}$. Because nothing explicitly depends of $\varphi$ it remains the same as at initial time. In Fig. 1, we plot radial velocity $\dot{r}$ in function of $u$. While the Fig. 2 shows the value of velocity $\dot{v}$.

Mode $J_{2}$. In this case we set $H=J_{2}(-i r) e^{-(u-5)^{2}} \sin (2 \varphi)$. In Fig. 3, we plot the radial velocity $\dot{r}$. In Fig. 4, we show the value of angle $\varphi$. We see that in angular direction we have displacement memory effect in contrary to the others where we have velocity memory effect.

Mode $J_{4}$. In this case we set $H=J_{4}(-i r) e^{-(u-5)^{2}} \sin (4 \varphi)$. In Fig. 5, we plot the radial velocity $\dot{r}$. In Fig. 6, we show the value of angle $\varphi$.

\section{Discussion}

We studied the geodesic motion of test particle in the presence of the pp wave with torsion. Our analysis discovered that particles, after the passage of the wave, show a combination of displacement and velocity memory effect. In the angular direction we discovered that $\mathrm{pp}$ waves induce displacement memory effect, for comparison velocity memory effect takes place for axial gravitational waves [29]. After the passage of axial wave burst particles rotate with constant angular velocity around the symmetry axis. 


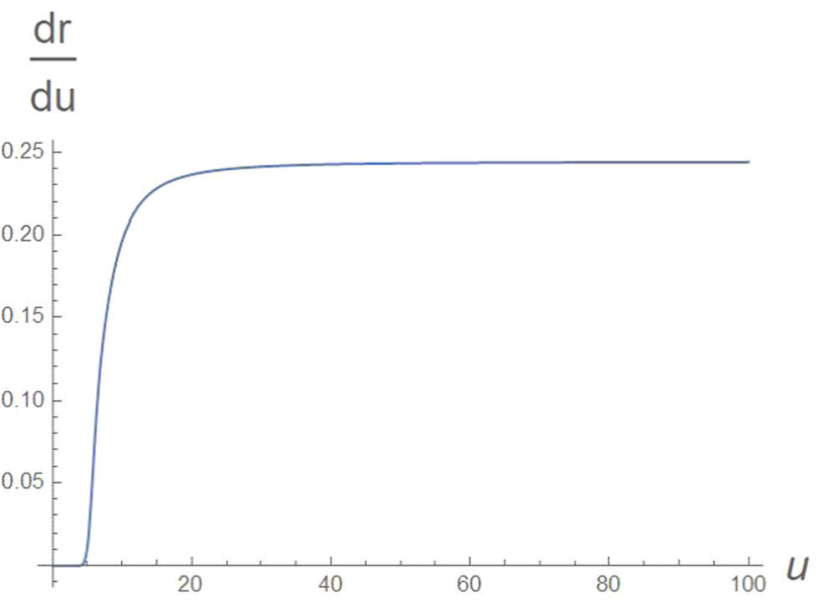

$$
\frac{d r}{d u}
$$

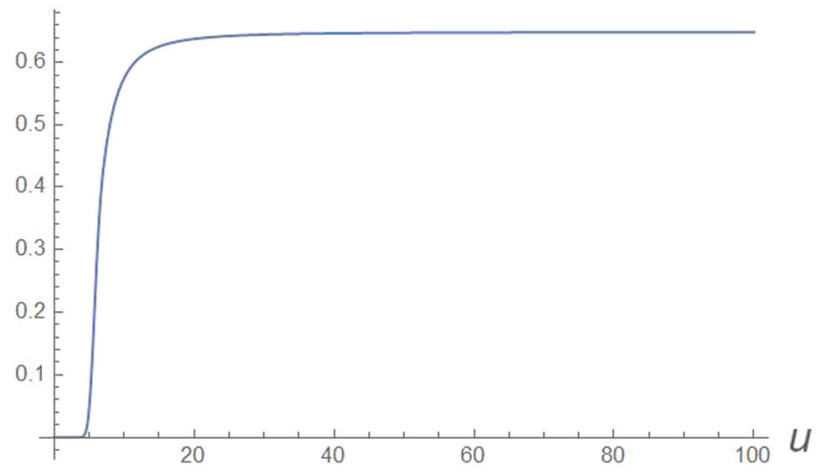

Fig. 3 The plot for the particle velocity $\dot{r}$ for $r[0]=1$ and $r[0]=2$ in both plots $\varphi[0]=0$
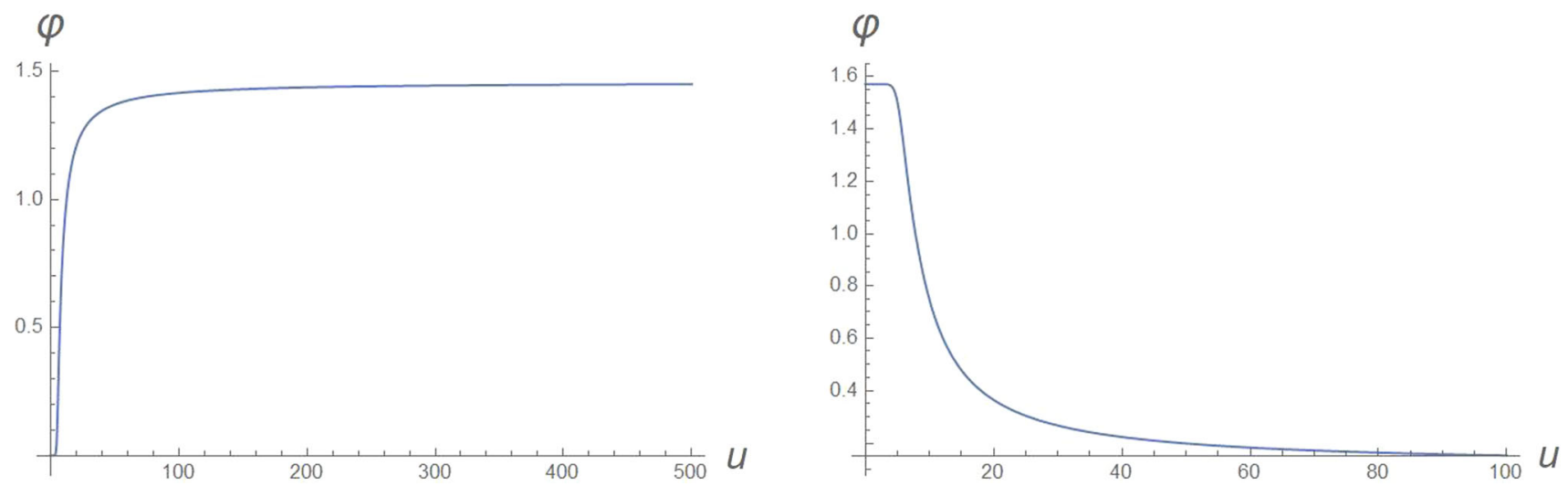

Fig. 4 The plot for the particles angular position $\varphi$ for $\varphi[0]=0$ and $\varphi[0]=\frac{\pi}{2}$ in both plots $r[0]=1$
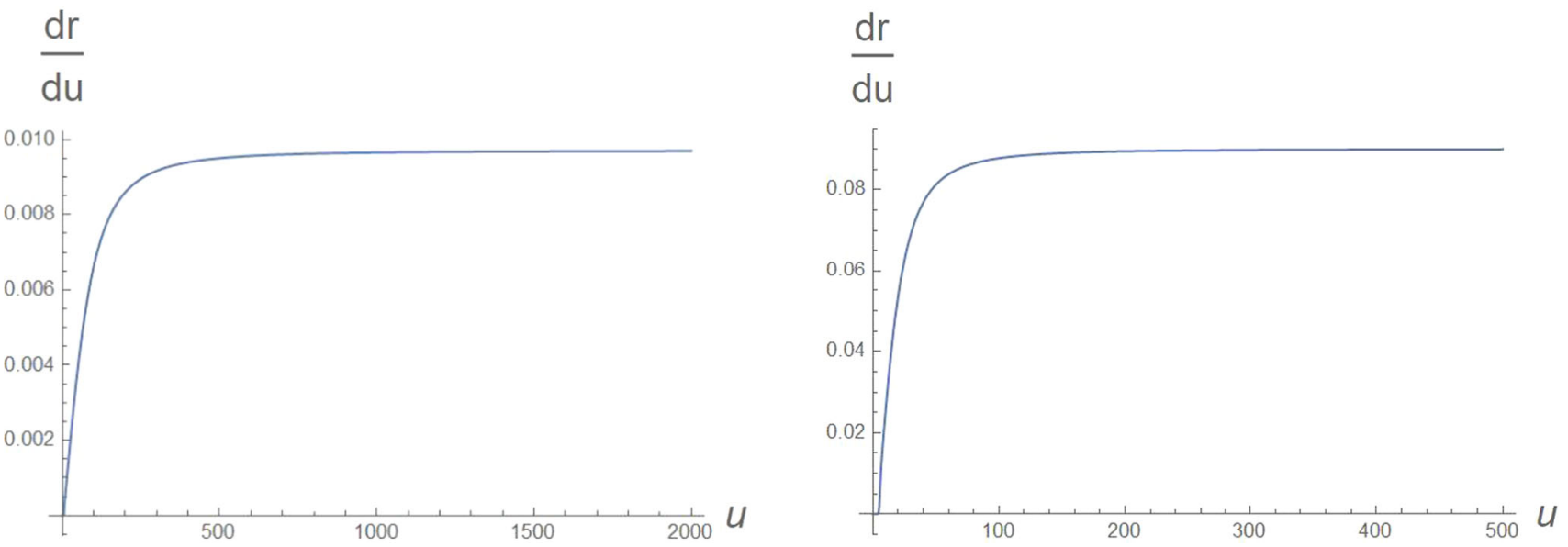

Fig. 5 The plot for the particle velocity $\dot{r}$ for $r[0]=1$ and $r[0]=2$ in both plots $\varphi[0]=0$ 

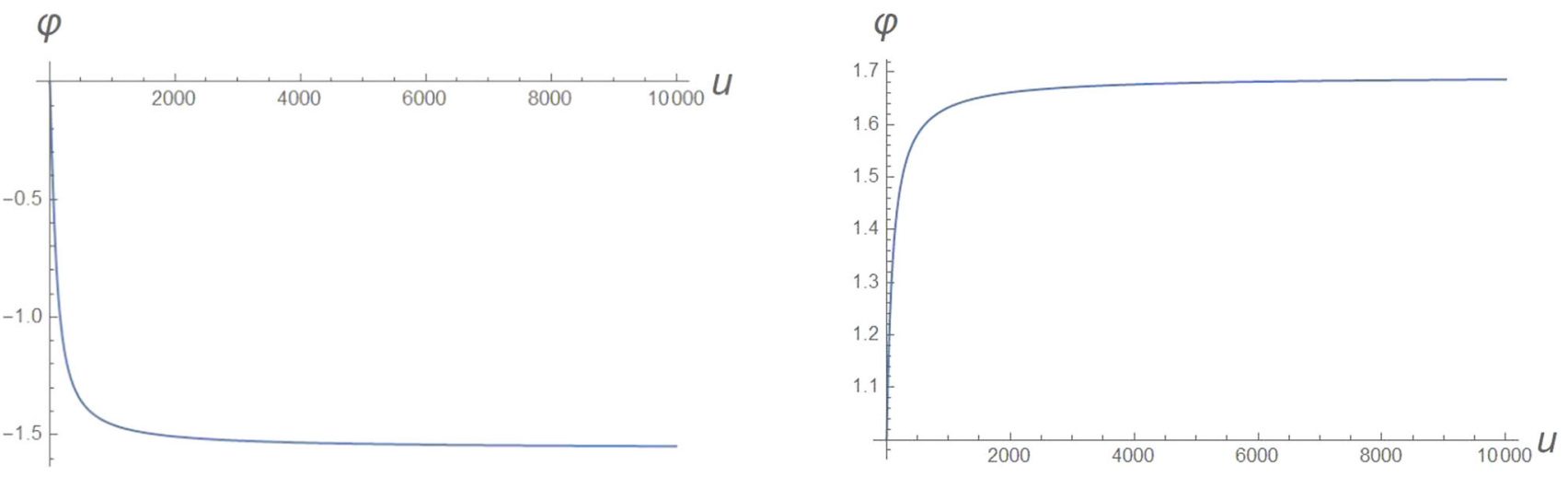

Fig. 6 The plot for the particles angular position $\varphi$ for $\varphi[0]=0$ and $\varphi[0]=1$ in both plots $r[0]=1$

Because we defined new variables $\mathrm{v}=m^{2} v$ and $r=m \rho$ our results have to be interpreted with taking into account the reasonable value of $m$. According to the CERN results we expect that possible mass of tordion is no less that $10 \mathrm{TeV}$

$m \geq 10 \mathrm{TeV}$.

This order of magnitude of the mass is equivalent to the length scale $\ell_{m}$

$\ell_{m} \approx 10^{-20} \mathrm{~m}$.

Due to the very large mass of tordion or, equivalently, very small length scale the physical values of $v$ and $\rho$ are very small and probably not observable. Fortunately $\varphi$ is insensitive to the value of the mass and offers a possible observable effect. We see from Figs. 4 and 6 that depending on the initial angular position the particle will be rotated by a different angle. Consequently, the particles initially set at some positions on a circle will be rotated relatively to each other. This is the possible experimental setup for the detection of torsion waves.

Acknowledgements This work was partially supported by the Serbian Ministry of Education, Science and Technological development.

Data Availability Statement This manuscript has no associated data or the data will not be deposited. [Authors' comment: All the data that we obtained are included in the article.]

Open Access This article is licensed under a Creative Commons Attribution 4.0 International License, which permits use, sharing, adaptation, distribution and reproduction in any medium or format, as long as you give appropriate credit to the original author(s) and the source, provide a link to the Creative Commons licence, and indicate if changes were made. The images or other third party material in this article are included in the article's Creative Commons licence, unless indicated otherwise in a credit line to the material. If material is not included in the article's Creative Commons licence and your intended use is not permitted by statutory regulation or exceeds the permitted use, you will need to obtain permission directly from the copyright holder. To view a copy of this licence, visit http://creativecomm ons.org/licenses/by/4.0/.

Funded by SCOAP ${ }^{3}$.

\section{A Solutions for the torsion functions $K_{\alpha}$}

In the spin- $2^{+}$sector, the torsion functions $K_{\alpha}$ can be determined (by using the condition $\Sigma=0$ ) from the equations:

$\partial_{y} \Theta+m^{2} K_{y}=0, \quad \partial_{z} \Theta+m^{2} K_{z}=0$.

Going over to polar coordinates,

$K_{y}=K_{\rho} \cos \varphi-\frac{K_{\varphi}}{\rho} \sin \varphi, \quad K_{z}=K_{\rho} \sin \varphi+\frac{K_{\varphi}}{\rho} \cos \varphi$,

the previous equations are transformed into

$K_{\rho}=-\frac{1}{m^{2}} \partial_{\rho} V, \quad K_{\varphi}=-\frac{1}{m^{2}} \partial_{\varphi} V$,

or equivalently, in terms of the Fourier modes,

$K_{\rho n}=-\frac{1}{m^{2}} \frac{p}{q} \partial_{\rho} \Theta_{n}, \quad K_{\varphi n}=-\frac{1}{m^{2}} n \Theta_{n}$,

where $K_{\varphi}=\sum_{n=1}^{\infty}\left(d_{n} e^{i n \varphi}+\bar{d}_{n} e^{-i n \varphi}\right)$ with $d_{n}=-i c_{n}$, and similarly for $K_{\rho}$.

\section{References}

1. Ya.. B. Zeldovich, A.G. Polnarev, Radiation of gravitational waves by a cluster of superdense stars. Astron. Zh. 51, 30 (1974)

2. Ya.. B. Zeldovich, A.G. Polnarev, Radiation of gravitational waves by a cluster of superdense stars. Sov. Astron. 18, 17 (1974)

3. V.B. Braginsky, L.P. Grishchuk, Kinematic resonance and the memory effect in free mass gravitational antennas. Zh. Eksp. Teor. Fiz. 89, 744 (1985)

4. V.B. Braginsky, L.P. Grishchuk, Kinematic resonance and the memory effect in free mass gravitational antennas. Sov. Phys. JETP 62, 427 (1985)

5. D. Christodoulou, Nonlinear nature of gravitation and gravitational wave experiments. Phys. Rev. Lett. 67, 1486 (1991)

6. K.S. Thorne, Gravitational-wave bursts with memory: The Christodoulou effect. Phys. Rev. D 45, 520 (1992)

7. H. Bondi, F.A.E. Pirani, Gravitational waves in general relativity III. Exact plane waves. Proc. Roy. Soc. Lond. A 251, 519-533 (1959) 
8. L.P. Grishchuk, A.G. Polnarev, Gravitational wave pulses with velocity coded memory. Sov. Phys. JETP 69, 653 (1989)

9. L..P. Grishchuk, A..G. Polnarev, Gravitational wave pulses with velocity coded memory. Zh. Eksp. Teor. Fiz. 96, 1153 (1989)

10. P.-M.Zhang, C. Duval, G.W. Gibbons, P.A. Horvathy, The Memory Effect for Plane Gravitational Waves. Phys. Lett. B 772, 743-746 (2017)

11. P.M.Zhang, C. Duval, G.W. Gibbons, P.A. Horvathy, Soft gravitons and the memory effect for plane gravitational waves. Phys. Rev. D 96(no.6), 064013 (2017)

12. P.M. Zhang, C. Duval, G.W. Gibbons, P.A. Horvathy, Velocity Memory Effect for Polarized Gravitational Waves. JCAP 1805(no.05), 030 (2018)

13. E. Kilicarslan, B. Tekin, Graviton mass and memory. Eur. Phys. J. C 79(no 2), 114 (2019)

14. I. Chakraborty, S. Kar, Geodesic congruences in exact plane wave spacetimes and the memory effect. Phys. Rev. D 101, 6 (2020)

15. B. Cvetković, D. Simić, Velocity memory effect without soft particles. Phys. Rev. D 101(2), 024006 (2020)

16. M. Blagojević, Gravitation and Gauge Symmetries (IoP Publishing, Bristol, 2002)

17. T. Ortín, Gravity and Strings (Cambridge University Press, Cambridge, 2004)

18. Yu.N. Obukhov, Poincaré gauge gravity: Selected topics. Int. J. Geom. Meth. Mod. Phys. 3, 95-138 (2006)

19. M. Blagojević, F.W. Hehl (eds.), Gauge Theories of Gravitation, A Reader with Commentaries (Imperial College Press, London, 2013)
20. M. Blagojević, B. Cvetković, Generalized $p p$ wavew in Poincaré gauge theory. Phys. Rev. D 95, 104018 (2017)

21. Yu.N. Obukhov, Generalized plane-fronted gravitational waves in any dimension. Phys. Rev. D 69, 024013 (2004)

22. K. Hayashi, T. Shirafuji, Gravity from Poincaré gauge theory of fundamental interactions. I. General formulation. Prog. Theor. Phys. 64, 866-882 (1980)

23. K. Hayashi, T. Shirafuji, Gravity from Poincaré gauge theory of fundamental interactions. IV. Mass and energy of particle spectrum. Prog. Theor. Phys. 64, 2222-2241 (1980)

24. E. Sezgin, P. van Nieuwenhuizen, New ghost-free gravity Lagrangians with propagating torsion. Phys. Rev. 21, 3269-3280 (1980)

25. D. Puetzfeld, Yuri N. Obukhov, Probing non-Riemannian spacetime geometry. Phys. Lett. A 372, 6711-6716 (2008)

26. P. Baekler, A spherically symmetric vacuum solution of the quadratic Poincare gauge field theory of gravitation with Newtonian and confinement potentials. Phys. Lett. 99(B), 329-332 (1981)

27. J.D. McCrea, P. Baekler, M. Gürses, A Kerr-like solution of the Poincaré gauge field equations. Nuovo Cimento B 99, 171-177 (1987)

28. Yu. N. Obukhov, Exact solutions in Poincaré gauge gravity theory. Universe 5, 127, 13 pages (2019)

29. W. Kulczycki, E. Malec, Axial gravitational waves in FLRW cosmology and memory effects. Phys. Rev. D 96(no.6), 063523 (2017) 